\title{
Transfer Function Identification from Phase Response Data
}

\author{
Luciano De Tommasi, Dirk Deschrijver and Tom Dhaene
}

This paper introduces an improved procedure for the identification of a transfer function from phase angle data with prescribed frequency variation. It is shown how a transfer function can be identified from phase response data samples, by fitting a normalized function with constant magnitude using the Vector Fitting algorithm. The presented approach is numerically robust and leads to more accurate results than conventional approaches.

\section{Introduction}

The synthesis of networks having prescribed phase characteristics is used for the design of feedback amplifier networks, symmetrical filter networks [1-2] and the design of low-pass filters with linear phase [3]. It is known from literature [4] that a transfer function can be determined from phase angle data by fitting the response of a tangent function using a polynomialbased Sanathanan-Koerner (SK) iteration. As shown in this paper, the reliability of this approach is sensitive to the dynamic behaviour of the tangent function, since it may contain a lot of sharp spikes which are difficult to approximate. To avoid this problem, a novel approach is presented that is based on least squares fitting of a proposed normalized function with constant magnitude by Vector Fitting (VF) [5-8]. Numerical examples illustrate the robustness of the proposed approach. It is also noted that the proposed method is computationally efficient, since it avoids the need for semidefinite programming which has been reported in more recent work [3].

\section{Transfer Function Identification via Tangent Approximation}

The problem of synthesizing a finite two-terminal network whose phase angle is a prescribed function of frequency was theoretically solved by Adler in 1948 [2]. In the following, such result is reviewed.

Let us consider the following transfer function:

$H(s)=\frac{a_{0}+a_{1} s+a_{2} s^{2}+\ldots+a_{m} s^{m}}{b_{0}+b_{1} s+b_{2} s^{2}+\ldots+b_{n} s^{n}}=\frac{P(s)}{Q(s)}$

where $m \leq n$ and the model coefficients $\left\{a_{i}\right\},\left\{b_{i}\right\}$ are real. Setting $s=j \omega$ in (1), the phase angle $\phi(\omega)$ of $H(j \omega)=|H(j \omega)| \exp (j \phi(\omega))$ is then given by:

$\tan \phi(\omega)=\left.\frac{\frac{1}{j} H_{o}(s)}{H_{e}(s)}\right|_{s=j \omega}$

where $H_{e}(s)$ and $H_{o}(s)$ are respectively, the even and odd part of $H(s)$ :

$H_{e}(s)=\frac{1}{2}[H(s)+H(-s)]=\frac{\frac{1}{2}[P(s) Q(-s)+P(-s) Q(s)]}{Q(s) Q(-s)} \equiv \frac{M(s)}{Q(s) Q(-s)}$, 
$H_{o}(s)=\frac{1}{2}[H(s)-H(-s)]=\frac{\frac{1}{2}[P(s) Q(-s)-P(-s) Q(s)]}{Q(s) Q(-s)} \equiv \frac{N(s)}{Q(s) Q(-s)}$.

Combining numerators of (3) and (4) yields:

$M(s)+N(s)=P(s) Q(-s)$,

which indicates that the zeros of the polynomial $M(s)+N(s)$ are both the zeros of $H(s)$ and its poles having opposite sign. Hence, the practical problem of determining poles and zeros of a transfer function $H(s)$, whose phase angle in the frequency domain $\phi(\omega)=\angle H(j \omega)$ approximates a set of phase response data $\left\{\omega_{k}, \phi^{\prime}\left(\omega_{k}\right)\right\}_{k=1 \ldots K}$, can be tackled computing the coefficients $\left\{c_{i}\right\},\left\{d_{i}\right\}$ of the following rational approximation:

$T\left(\omega_{k}\right)=\frac{\tan \phi\left(\omega_{k}\right)}{\omega_{k}}=\frac{N\left(j \omega_{k}\right)}{j \omega_{k} M\left(j \omega_{k}\right)}=\frac{c_{0}+c_{1} \omega_{k}^{2}+\ldots+c_{p} \omega_{k}^{2 p}}{1+d_{1} \omega_{k}^{2}+\ldots+d_{p} \omega_{k}^{2 p}} \approx \frac{\tan \phi^{\prime}\left(\omega_{k}\right)}{\omega_{k}}$,

where $2 p+1=m+n$, and afterwards the roots of $M(s)+N(s)=0$. Several transfer functions can be constructed which approximate a given phase response, depending on how the zeros of $M(s)+N(s)$ are assigned to $P(s)$ and $Q(-s)$. If it is required to obtain a minimum phase shift function, then all poles and zeros must be located within the left-half plane. In that case, the roots of $M(s)+N(s)=0$, belonging to the right half plane must be assigned to $Q(-s)$, whereas the roots belonging to the left half plane must be assigned to $P(s)$. Once the roots of $P(s)$ and $Q(s)$ are known, the transfer function is completely determined except for an arbitrary constant multiplier which has no influence upon the phase angle.

Adler used rational interpolation in [2], which means that the left hand side exactly equals the left hand side in (6). Later on, Jong [4] introduced the rational approximation in (6) using a least-squares approach based on polynomial-based Sanathanan-Koerner (SK) algorithm [6]. However, the rational interpolation/approximation (6) is numerically difficult to achieve because of the large peaks of the tangent function [2]. Moreover, since a dense sample distribution is required in order to describe accurately a peak, a non-uniform sample distribution is advisable to avoid oversampling in smooth regions of the tangent function [2].

In section 3, a new approach, numerically more robust than tangent approximation, is proposed. Such approach has been validated on several numerical examples. Due to space limitation, only two examples are shown in the paper (sections 4 and 5).

It is worth to remark that, when phase angle data samples come from a physically realizable system (i.e. a system where $m \leq n)$, the number of right-half plane zeros of $M(s)+N(s)$ cannot be less than half of the total number of zeros. On the other hand, provided that the approximation (6) is sufficiently accurate, a number of right half plane zeros less than $\frac{m+n}{2}$ indicates that the given phase angle data samples cannot be approximated with any physically realizable (stable) 
systems of order at most $m+n$.

Finally, we mention that the polynomial $M(s)+N(s)$ never owns any imaginary roots, because these cancel out in the ratio $\frac{N}{M}$. A comprehensive discussion is omitted in this paper; the interested reader can refer to [1].

\section{Transfer Function Identification via the New Proposed Approach}

The identification of the transfer function $H(s)$ whose phase angle in the frequency domain $\phi(\omega)=\angle H(j \omega)$ approximates a set of phase response data $\left\{\omega_{k}, \phi^{\prime}\left(\omega_{k}\right)\right\}_{k=1 \ldots K}$, can be achieved by avoiding the rational approximation (6).

Let us define the function:

$$
\Phi(s)=\frac{M(s)-N(s)}{M(s)+N(s)}
$$

whose poles are the roots of $P(s) Q(-s)$, see equation (5). Therefore, the poles and zeros of transfer function $H(s)$ can be identified by calculating the poles of $\Phi$. Such calculation is pursued in the frequency domain $(s=j \omega)$. It results from (2),(3),(4):

$\Phi(j \omega)=\frac{1-j \tan \phi(\omega)}{1+j \tan \phi(\omega)}$

hence the VF macromodeling algorithm [5] is applied to solve the following pole identification problem (where the unknowns are $\left.\left\{p_{i}\right\}\right)$ :

$\Phi\left(j \omega_{k}\right)=r_{0}+\sum_{i=1}^{n+m} \frac{r_{i}}{j \omega_{k}-p_{i}} \approx \Phi^{\prime}\left(j \omega_{k}\right)=\frac{1-j \tan \phi^{\prime}\left(\omega_{k}\right)}{1+j \tan \phi^{\prime}\left(\omega_{k}\right)}$.

The VF algorithm solves a rational approximation problem in two steps, respectively named pole identification (which gives the $\left\{p_{i}\right\}$ ) and residue identification (which gives the $\left\{r_{i}\right\}$ ). The pole identification solely involves the solution of a linear system of equations and of an associated eigenvalue problem. The residue identification consists only of a linear system of equations. Both systems of equations are overdetermined when the number of frequency samples is greater than the number of model parameters. In such a case the solution is pursued in the least-squares sense. The reader is referred to [5-8] for further details. Since $\left\{p_{i}\right\}$ contains all required information, the identification of residues $\left\{r_{i}\right\}$ can be skipped in (9).

It is worth to note that the function $\Phi(j \omega)$ is much smoother than the tangent, since it has a constant magnitude. Hence, the computation of the rational approximation (9) results in accurate poles and zeros based on a uniform sample distribution. At the same time, it is well-known from [7] that VF algorithm is numerically more robust than the polynomial SK iteration. 


\section{Example 1: Comparison between Tangent Rational Approximation Approach and the Proposed Approach}

The proposed method has been applied to identify the transfer function of a RLC filter, whose phase angle approximates the phase angle of the filter provided below (for $s=j \omega)$ :

$$
\begin{aligned}
& Z^{\prime}(s)=-\frac{3000}{s+4500}-\frac{83000}{s+41000}-\frac{5-j 7000}{s+100-j 5000}-\frac{5+j 7000}{s+100+j 5000}+ \\
& -\frac{20-j 18000}{s+120-j 15000}-\frac{20+j 18000}{s+120+j 15000}+\frac{6000+j 45000}{s+3000-j 35000}+\frac{6000-j 45000}{s+3000+j 35000}+ \\
& +\frac{40+j 60000}{s+200-j 45000}+\frac{40-j 60000}{s+200+j 45000}+\frac{90+j 10000}{s+1500-j 45000}+\frac{90-j 10000}{s+1500+j 45000}+ \\
& +\frac{50000+j 80000}{s+500-j 70000}+\frac{50000-j 80000}{s+500+j 70000}+\frac{1000+j 45000}{s+1000-j 73000}+\frac{1000-j 45000}{s+1000+j 73000}+ \\
& -\frac{5000-j 92000}{s+2000-j 90000}-\frac{5000+j 92000}{s+2000+j 90000}+0.2
\end{aligned}
$$

The corresponding frequency domain phase response data $\angle Z^{\prime}\left(j \omega_{k}\right)$ have been evaluated over $K=200$ angular frequency points $\left\{\omega_{k}\right\}_{k=1 \ldots K}$, uniformly spaced throughout the interval $10-100000 \mathrm{rad} / \mathrm{s}$. The phase angle $\angle Z^{\prime}\left(j \omega_{k}\right)$ is contaminated by Gaussian noise $n\left(\omega_{k}\right)$, with mean $\mu=0$ and standard deviation $\sigma=0.001$ :

$$
\phi^{\prime}\left(\omega_{k}\right)=\angle Z^{\prime}\left(j \omega_{k}\right)+n\left(\omega_{k}\right)
$$

First, as earlier proposed in [1],[4], we tried to calculate the rational approximation of the tangent function (6) with $p=14$. It is found that the sharp spikes of $\frac{\tan \phi^{\prime}\left(\omega_{k}\right)}{\omega_{k}}$ are difficult to approximate with a decent accuracy, as shown in Fig. 1. Therefore, the newly proposed method is applied to identify the function $\Phi(j \omega)(9)$ with $m+n=28$. Figure 2 shows that an excellent correspondence is obtained between the phase angle of the reference function $\Phi^{\prime}(j \omega)$ and the direct rational approximation $\Phi(j \omega)$ (proposed approach). Although not required by the procedure described in section 2 , a rational approximation $\Phi_{T}(j \omega)$ of $\Phi^{\prime}(j \omega)$ is also computed from the rational approximation $T(\omega)(6)$ :

$\Phi_{T}\left(j \omega_{k}\right)=\frac{1-j \omega_{k} T\left(\omega_{k}\right)}{1+j \omega_{k} T\left(\omega_{k}\right)} \approx \Phi^{\prime}\left(j \omega_{k}\right)=\frac{1-j \tan \phi^{\prime}\left(\omega_{k}\right)}{1+j \tan \phi^{\prime}\left(\omega_{k}\right)}$,

This allows a direct comparison between the proposed approach and tangent approximation. Figure 2 shows that $\Phi_{T}(j \omega)$ is less accurate than $\Phi(j \omega)$. In particular, the inaccuracy found in the approximation $T(\omega)$ around $70 \mathrm{krad} / \mathrm{s}(\mathrm{see}$ fig. 1) is clearly reflected in $\Phi_{T}(j \omega)$.

A transfer function $Z(s)=\frac{P(s)}{Q(s)}$ such that $\angle Z(j \omega) \approx Z^{\prime}(j \omega)$ can now easily be reconstructed, by assigning the poles of $\Phi$ to $P(s)$ and $Q(-s)$. With both tangent approximation and proposed approach, the same procedure is undertaken. The first fifteen poles of $\Phi$ (proposed approach) and zeros of $M(s)+N(s)$ (tangent approximation) with positive real part are assigned to $Q(-s)$, the others are assigned to $P(s)$. Different assignments would lead to different functions $Z(s)$ which all have the same phase angle, but differ for the magnitude response. Since the poles of $\Phi$ are identified with a very high 
accuracy, an excellent agreement between the phase angle of $Z^{\prime}(j \omega)$ and the phase angle of $Z(j \omega)$ can be observed in Fig. 3. On the other hand, since the tangent approximation (6) is inaccurate (Fig. 1 and Fig. 2) the agreement between the final approximation $Z_{T}(j \omega)$ obtained with (6) and the reference function $Z^{\prime}(j \omega)$ is inaccurate as well.

\section{Example 2: Synthesis problem of a linear phase low-pass filter}

In this section the proposed approach is used to construct a low pass filter with a linear phase for low frequencies. The filter is then compared with two standard Bessel filters.

Low-pass filters with linear phase are desired because they do not distort the signal, but only introduce a delay. Since Finite Impulse Response (FIR) filters can be easily constructed in the discrete time, it was proposed in [3] to derive a continuous time filter by fitting a rational transfer function $G_{a}(s)$ to frequency samples $G\left(j \omega_{k}\right)$ generated by a discrete time linear phase FIR filter. The same procedure is followed here, but the new method introduced in this paper is applied instead of the one presented in [3]. Compared to [3], the new proposed approach is much simpler and computationally less expensive. It only requires the solution of a linear system of equations and of an eigenvalue problem (VF pole identification), whereas [3] formulates a semidefinite programming problem.

Let us consider the discrete time linear phase FIR filter:

$G(j \omega)=\sum_{l=0}^{5} c_{l} e^{-l \pi j \omega}$

with cut-off frequency $0.5 \mathrm{rad} / \mathrm{s}$ and $\left\{\omega_{k}\right\}=\{0,0.01, \ldots, 0.7\}$. The coefficients $\left\{c_{l}\right\}$ can be obtained by the MATLAB command firl $(5,0.5)$. The transfer function $G_{a}(s)$ of a fifth-order continuous time filter will be identified from $\angle G\left(j \omega_{k}\right)$. First, the samples $\left\{\Phi^{\prime}\left(j \omega_{k}\right)\right\}(9)$ are evaluated with $\phi^{\prime}\left(\omega_{k}\right)=\angle G\left(j \omega_{k}\right)$. Then, the rational approximation (9) is computed with $n+m=5$. Since all the poles $\left\{p_{i}\right\}$ of $\Phi(s)$ lie in the right-half plane, they can all be assigned to $Q(-s)$ ( $m=0, n=5$ ) resulting in a stable system. This is indeed the best solution, since it results in a filter with the strongest attenuation possible in its stop-band. The final result is:

$G_{a}(s)=\frac{0.0155}{(s+0.2428)(s+0.2313+0.3161 j)(s+0.2313-0.3161 j)(s+0.1814+0.6182 j)(s+0.1814-0.6182 j)}$.

Figure 4 shows that the phase angle of $G_{a}(j \omega)$ matches the phase angle of $G(j \omega)$ up to $0.7 \mathrm{rad} / \mathrm{s}$. Figure 5 shows that the cut-off frequency is close to $0.5 \mathrm{rad} / \mathrm{s}$. Like in [3], we have compared the filter $G_{a}(s)$ with two different fifth-order Bessel filters, $G_{b 1}$ and $G_{b 2}$, respectively obtained by the MATLAB command besself $(5,0.5)$ and besself $(5,0.7)$. The filter $G_{b 1}(j \omega)$ matches the phase angle of $G(j \omega)$ and $G_{a}(j \omega)$ up to $0.5 \mathrm{rad} / \mathrm{s}$, but has a lower cut-off frequency. On the other hand, the filter $G_{b 2}(j \omega)$, exhibits a linear phase angle up to $0.7 \mathrm{rad} / \mathrm{s}$, but with less phase lag than $G(j \omega)$ and $G_{a}(j \omega)$ and with a higher cut-off frequency. Hence, we conclude that $G_{a}$ is not a standard Bessel filter. 


\section{Conclusions}

A new procedure for the transfer function identification from phase response data is presented. The VF technique is used to approximate a normalized function of the phase angle, allowing a straightforward and accurate identification of poles and zeros of the transfer function. The effectiveness of this approach has been shown on a practical filter synthesis problem.

\section{Acknowledgments}

This work is part of the O-MOORE-NICE! project as supported by the European Commission through the Marie Curie Actions of its Sixth Program under contract number MTKI-CT-2006-042477. The work is also supported by the Fund for Scientific Research in Flanders (FWO Vlaanderen).

\section{References}

[1] Guillemin, E. A., 'Synthesis of Passive Networks', John Wiley and Sons, 1957.

[2] Adler, A., 'Synthesis of a Finite Two-Terminal Network whose Phase Angle is a Prescribed Function of Frequency with Application to the Design of Symmetrical Networks', Massachussetts Institute of Technology, 1948, available on-line at: http://dspace.mit.edu/handle/1721.1/12619.

[3] Sandberg, H., Lanzon, A., Anderson, B.D.O., 'Model approximation using magnitude and phase criteria: Implications for model reduction and system identification', Int. J. Robust Nonlinear Control, no. 17, pp. 435-461, 2007.

[4] Jong, M.T. , 'Determination of a Transfer Function from Phase Response Data', Proceedings of IEEE, vol. 67, no. 4, pp. 683-684, April 1979.

[5] Gustavsen, B. , 'Rational Approximation of Frequency Domain Responses by Vector Fitting', IEEE Trans. on Power Delivery, vol. 14, no. 3, pp. 1052-1061, July 1999.

[6] Hendrickx, W., Dhaene, T. , 'A Discussion of "Rational Approximation of Frequency Domain Responses by Vector Fitting', IEEE Trans. on Power Delivery, vol. 21, no. 1, pp. 441-443, February 2006.

[7] D. Deschrijver. B. Gustavsen, T. Dhaene, 'Advancements in Iterative Methods for Rational Approximation in the Frequency Domain', IEEE Trans. on Power Delivery, vol. 22, no. 3, pp. 1633-1642, July 2007.

[8] Gustavsen, B., 'Computer Code for Rational Approximation of Frequency Dependent Admittance Matrices', IEEE Trans. on Power Delivery, vol. 17, no. 4, pp. 1093-1098, October 2002. 


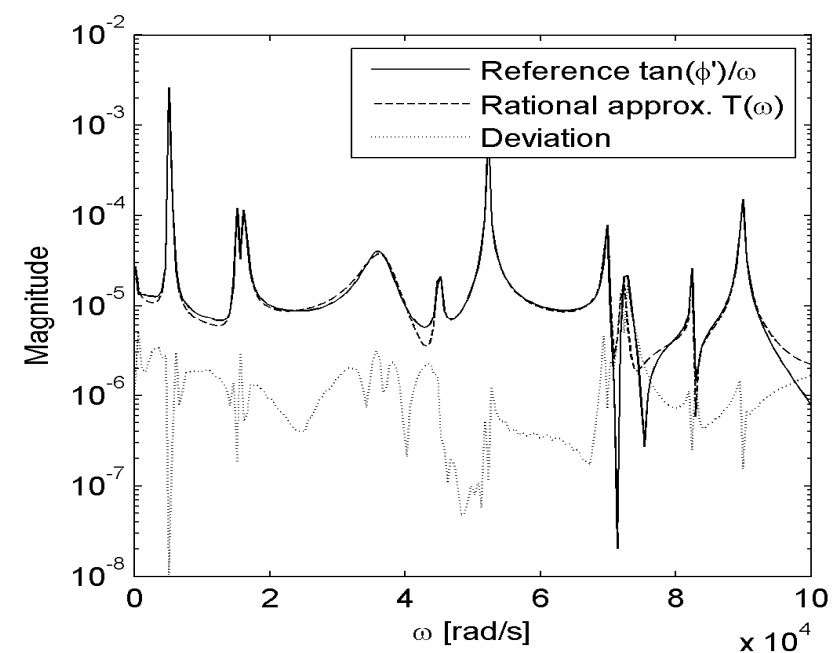

Figure 1. Example 1: rational approximation $T(\omega)$ of (6), using ten Vector Fitting iterations.

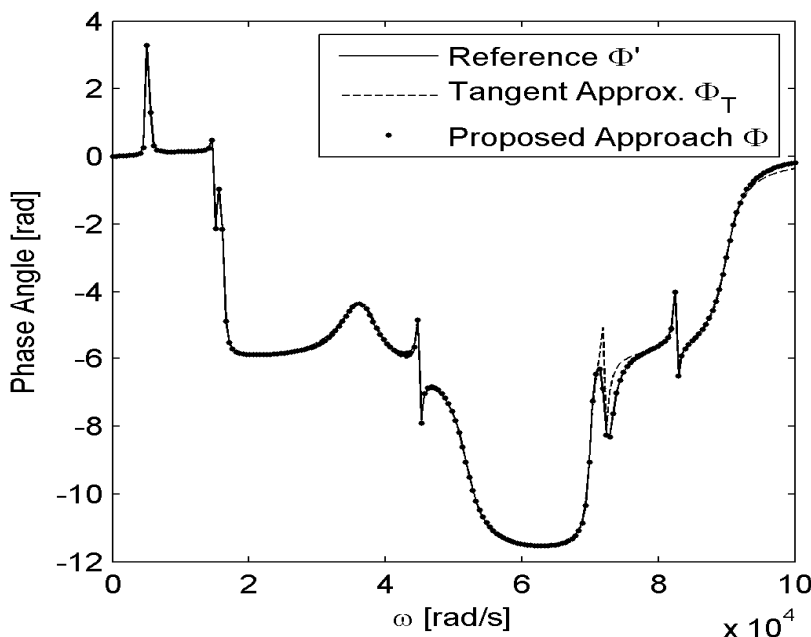

Figure 2. Example 1: proposed approach, direct rational approximation of $\Phi^{\prime}$ (9) (using ten Vector Fitting iterations) compared with $\Phi_{T}(12)$.

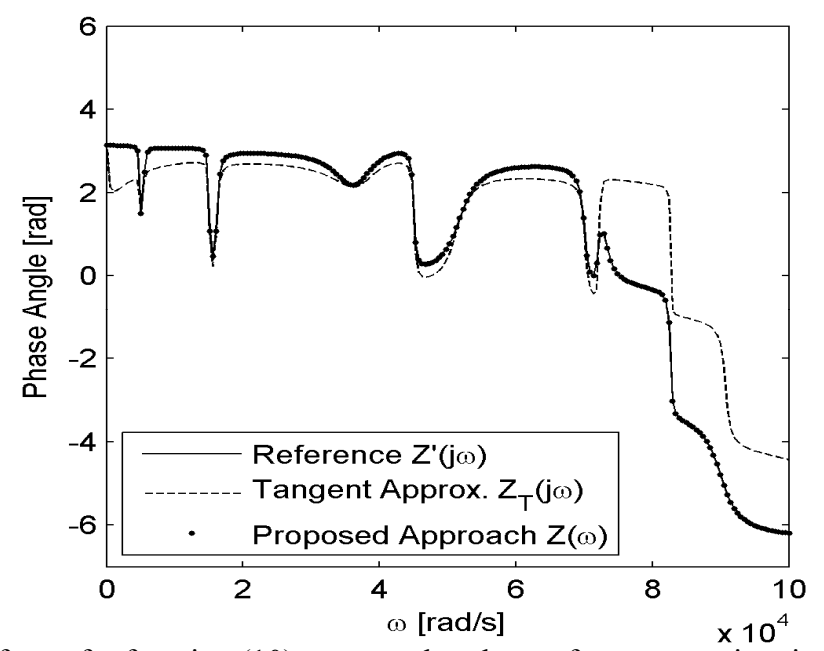

Figure 3. Example 1: phase angle of transfer function (10) compared to those of two approximations obtained with the proposed approach and the tangent approximation approach.

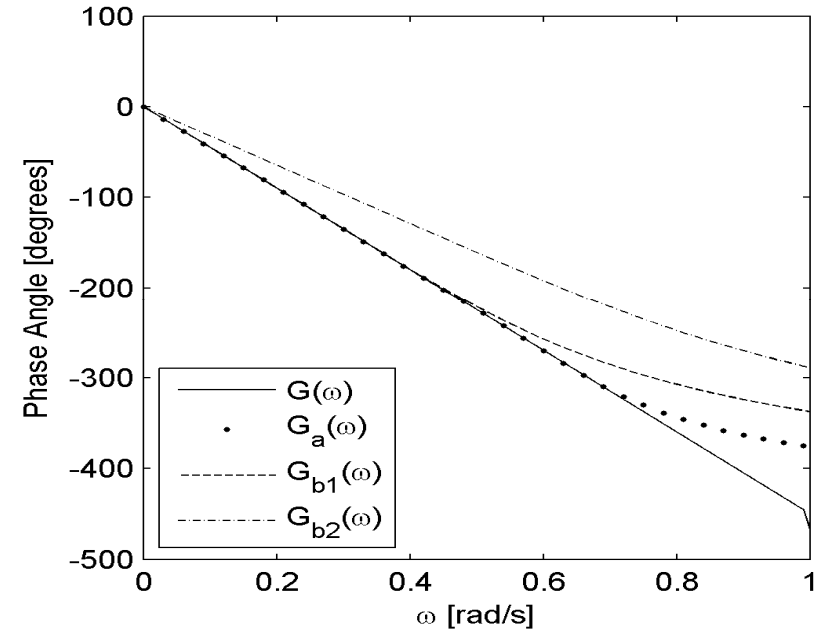

Figure 4. Example 2: phase angle of a linear phase filter $G_{a}$ identified with proposed approach, compared to a couple of standard Bessel filters $G_{b 1}$ and $G_{b 2}$.

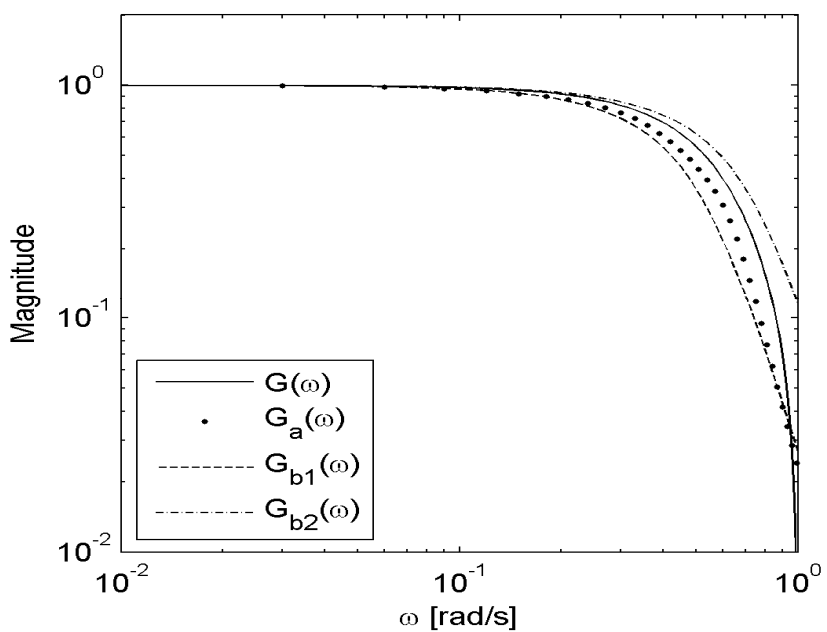

Figure 5. Example 2: magnitude of a linear phase filter $G_{a}$ identified with proposed approach, compared to a couple of standard Bessel filters $G_{b 1}$ and $G_{b 2}$. 


\section{$\underline{\text { Author Affiliations }}$}

Dr. Luciano De Tommasi (a)(c), Dr. Dirk Deschrijver (b) and Prof. Tom Dhaene (b)(c)

(a) NXP Semiconductors, High Tech Campus 37, 5656 AE Eindhoven, The Netherlands

(b) Ghent University - IBBT, Sint Pietersnieuwstraat 41, 9000 Ghent, Belgium

(c) University of Antwerp, Middelheimlaan 1, 2020 Antwerpen, Belgium

Email : 1uciano.detommasi@ua.ac.be, dirk.deschrijver@ugent.be, tom.dhaene@ugent.be 